\title{
A GENERALIZATION OF COMMUTATIVITY THEOREM FOR RINGS*
}

\author{
BY
}

\section{JUNCHAO WEI and ZHIYONG FAN}

\begin{abstract}
Let $R$ be a ring with an identity and for each $x \in S N(R)=\{x \in R \mid x \notin$ $N(R)\}$ and $y \in R,(x y)^{k}=x^{k} y^{k}$ for three consecutive positive integers $k$. It is shown in this note that $R$ is a commutative ring, which generalizes the known theorem belonging to LigH and RichOuX.
\end{abstract}

Mathematics Subject Classification 2010: 16A30, 16A50, 16E50, 16D30.

Key words: commutative rings, nilpotent elements, positive integers.

\section{Introduction}

Starting from 1950, the topic of commutativity of associative rings has been studied by many authors. In [2], PINTER-LUCKE introduced in details these works.

In [1], it is shown that if $R$ is a ring with an identity and for each $x, y \in R,(x y)^{k}=x^{k} y^{k}$ for three consecutive positive integers $k$, then $R$ is a commutative ring. The purpose of this note is to generalize this result.

Throughout this article, all rings considered are associative with an identity, the symbols $Z(R)$ and $N(R)$ will stand respectively for the center of $R$ and the set of all nilpotent elements. Let $R$ be a ring and write $S N(R)=\{x \in R \mid x \notin N(R)\}$.

\footnotetext{
*Project supported by the Foundation of Natural Science of China (11171291, 10771182), the Scientific Research Foundation of Graduate School of Jiangsu Province (CX09B_309Z) and Natural Science Fund for Colleges and Universities in Jiangsu Province(11KJB110019).
} 
Theorem 1.1. If $R$ is a ring satisfying $(x y)^{k}=x^{k} y^{k}$ for each $x \in$ $S N(R), y \in R$ and $k=n, n+1, n+2$, where $n$ is a positive integer, then $R$ is commutative.

Proof. Let $x, y \in R$. If $x \in N(R)$, then $1+x \in S N(R)$. By hypothesis, we have the following equation for $k=n, n+1, n+2$

$$
((1+x) y)^{k}=(1+x)^{k} y^{k}
$$

Since $1+x$ is invertible, simplify, to get

$$
\begin{aligned}
& y((1+x) y)^{n-1}=(1+x)^{n-1} y^{n}, \\
& y((1+x) y)^{n}=(1+x)^{n} y^{n+1}, \\
& y((1+x) y)^{n+1}=(1+x)^{n+1} y^{n+2} .
\end{aligned}
$$

Multiply (1.2) on the right by $(1+x) y$, keeping in mind the identity (1.3), it follows that

$$
y^{n} x y=x y^{n+1}
$$

Similarly, using (1.3) and (1.4), one gets

$$
y^{n+1} x y=x y^{n+2} .
$$

Multiply (1.5) on the left by $y$, from the identity (1.6), eventually one gets

$$
(y x-x y) y^{n+1}=0 .
$$

If $x \notin N(R)$, then $x \in S N(R)$, by hypothesis, for $k=n, n+1, n+2$, one gets

$$
(x y)^{k}=x^{k} y^{k} .
$$

If $1+x \in N(R)$, then $x$ is invertible, hence one obtains

$$
\begin{aligned}
& y(x y)^{n-1}=x^{n-1} y^{n}, \\
& y(x y)^{n}=x^{n} y^{n+1}, \\
& y(x y)^{n+1}=x^{n+1} y^{n+2} .
\end{aligned}
$$

Using the equations (1.9)-(1.11), employing the same technique used to get (1.7) from (1.2)-(1.4), one also obtains

$$
(y x-x y) y^{n+1}=0 .
$$


If $1+x \notin N(R)$, then by hypothesis, one gets

$$
\begin{aligned}
& ((1+x) y)^{n}=(1+x)^{n} y^{n}, \\
& ((1+x) y)^{n+1}=(1+x)^{n+1} y^{n+1}, \\
& ((1+x) y)^{n+2}=(1+x)^{n+2} y^{n+2} .
\end{aligned}
$$

Multiply (1.13) on the right by $(1+x) y$ and combine (1.14), one obtains

$$
(1+x)^{n}\left(y^{n} x-x y^{n}\right) y=0 .
$$

Similarly, by the equations (1.14) and (1.15), it follows that

$$
(1+x)^{n+1}\left(y^{n+1} x-x y^{n+1}\right) y=0 .
$$

Similarly, the equation (1.8) implies the following identities

$$
\begin{aligned}
& x^{n}\left(y^{n} x-x y^{n}\right) y=0, \\
& x^{n+1}\left(y^{n+1} x-x y^{n+1}\right) y=0 .
\end{aligned}
$$

Multiply (1.16) on the left by $x^{n-1}$ and expand $(1+x)^{n}$ by the binomial theorem, keeping in mind the identity (1.18); it follows that

$$
x^{n-1}\left(y^{n} x-x y^{n}\right) y=0 .
$$

Multiply (1.16) on the left by $x^{n-2}$ and expand $(1+x)^{n}$, keeping in mind the identity (1.18) and (1.20); it follows that

$$
x^{n-2}\left(y^{n} x-x y^{n}\right) y=0
$$

continue the above process, eventually one gets

$$
y^{n} x y=x y^{n+1} .
$$

Similarly, by the equations (1.17) and (1.19), one can obtain

$$
y^{n+1} x y=x y^{n+2} .
$$

This gives $y x y^{n+1}=y^{n+1} x y=x y^{n+2}$, that is

$$
(y x-x y) y^{n+1}=0 .
$$


Combine the equations (1.7), (1.12) and (1.24), one obtains that for each $x, y \in R$, the following identity holds

$$
(y x-x y) y^{n+1}=0 .
$$

Now apply the same technique used to get (1.22) from (1.16) and (1.18) by substituting $y+1$ for $y$ in (1.25), we then have

$$
x y=y x .
$$

Thus $R$ is commutative.

Corollary 1.2 ([1]). If $R$ is a ring satisfying the identities $(x y)^{k}=$ $x^{k} y^{k}, k=n, n+1, n+2$, where $n$ is a position integer, then $R$ is commutative.

\section{REFERENCES}

1. Ligh, S.; Richoux, A. - A commutativity theorem for rings, Bull. Austral. Math. Soc., 16 (1977), 75-77.

2. Pinter-Lucke, J. - Commutativity conditions for rings: 1950-2005, Expo. Math., 25 (2007), 165-174.

Received: 27.II.2012

School of Mathematics,

Accepted: 18.I.2013

Yangzhou University, Yangzhou, 225002,

P.R. CHINA

jcweiyz@126.com 